\title{
The Blue Straggler Population of the Globular Cluster M5: Comparison with M3
}

\author{
Steven R. Warren, Eric L. Sandquist \\ Department of Astronomy, San Diego State University, San Diego, CA \\ 92128;warren@sciences.sdsu.edu;erics@sciences.sdsu.edu \\ and \\ Michael Bolte \\ UCO/Lick Observatory, University of California, Santa Cruz, CA 95064;bolte@ucolick.org
}

\begin{abstract}
We have surveyed the blue straggler star population of the Galactic globular cluster M5 using high-resolution images of the core along with wide-field ground-based images reaching to more than 19 core radii. To gauge M5's relative efficiency of producing stragglers, we compared our sample to five studies of other globular clusters (mainly Ferraro et al. 1997b; Ferraro et al. 2003; and Piotto et al. 2004). Using a "bright" sample selected in the same way as Ferraro et al. (1997b), we found a bimodal radial distribution similar to those found in three other luminous clusters. When the radial distributions for different clusters are scaled using the core radius, there is good cluster-to-cluster agreement in the size of the core straggler sample and the center of the "zone of avoidance". However, M5 has the smallest fraction of stragglers in the zone of avoidance of any of the clusters measured to date, and its zone of avoidance appears to be wider (in $\left.r / r_{c}\right)$ than that of M3, which has a very similar surface brightness profile. Both of these facts indicate that M5's straggler population has dynamically evolved to a larger extent than M3. Using an ultraviolet sample from Hubble Space Telescope selected in the same way as Ferraro et al. (2003) and Ferraro et al. (2004), we find that the frequency of blue stragglers in M5 is lower than all but two of the clusters examined. We also identified seven bright blue stragglers that were previously misidentified as HB stars by Sandquist et al. (2004). These bright stragglers are most likely the result of stellar collisions involving binary stars.
\end{abstract}

Subject headings: blue stragglers — globular clusters: general — globular clusters: individual (M5, M3) 


\section{Introduction}

Blue straggler stars (BSSs) were first identified as stars brighter and bluer than the main-sequence turnoff in the cluster M3 by Sandage (1953). Since then they have been found in all globular clusters that have been surveyed adequately and in many open clusters. The current leading explanations for the production of BSSs involve mass transfer within a binary system or the merger of two stars in a binary, and stellar collisions.

The rate of stellar collisions within a cluster depends on its current structural characteristics (notably, central density and velocity dispersion), and on the collision cross sections of the interacting objects. The binary star population in a cluster can play a large role in BSS formation. In addition to evolution of a binary via mass-transfer and merger into a BSS, binaries have an enhanced collision rate for interaction with other stars. BSSs, being much like newly-created main sequence stars, are also likely to have lifetimes that are comparable to or larger than the timescales on which a cluster dynamically evolves. The stragglers are thus an easily accessible population of stars that can give us insight into a cluster's dynamical past and the importance of its present structural characteristics.

A large amount of effort has recently been put into seeking a global view of how BSS populations vary from cluster to cluster. Piotto et al. (2004) cataloged almost 3000 BSSs in the cores of 56 globular clusters based on Hubble Space Telescope (HST) observations, and found a significant anticorrelation between the relative frequency of BSS and total cluster luminosity, and little or no correlation with other cluster parameters. Although the trend with luminosity is strong, there is a significant amount of scatter (perhaps by as much as an order of magnitude) around the trend.

In M3, Ferraro et al. (1993) discovered a bimodal distribution of BSSs with respect to radius, with separate groups of core and envelope stragglers (see also Ferraro et al. 1997b). Since then, such distributions have been identified in other clusters (M55, Zaggia et al. 1997; 47 Tuc, Ferraro et al. 2004; NGC 6752, Sabbi et al. 2004). However, in the low-luminosity cluster Palomar 13 (Clark et al. 2004), the frequency of BSSs does not increase in the outskirts of the cluster.

The small number of studies of the complete radial distribution of BSSs has led us to examine the cluster M5. M5 has structural characteristics that are currently very similar to M3 (see Table 1). As such, M5 provides an interesting test of whether clusters with similar structural properties must necessarily have similar straggler populations.

In $\S 2$, we present the datasets that were used in the identification of stragglers in M5. In $\S 3$, we present the total sample of stragglers, and in $\S 4$, we compare the M5 sample to those from other globular clusters. In $\S 5$, we discuss the comparison population of horizontal 
branch (HB) stars. Finally, we discuss the size of the straggler population and its radial distribution in $\S 6$.

\section{Observations and Data Reduction}

The photometric data we used in this study were derived from images taken using several ground-based telescopes and HST. Wide-field images in $B, V$, and $I$ were taken at the Cerro Tololo Interamerican Observatory (CTIO) $4 \mathrm{~m}$ telescope, and their reduction has already been described in Sandquist et al. (1996). The images were centered on the cluster core and covered square fields 16.3 on a side. We used additional higher resolution images of the cluster core from the Canada-France-Hawaii $3.5 \mathrm{~m}$ Telescope (CFHT) in $B$ and $I$. These images have also been discussed previously (Sandquist et al. 1996, 2004). The field covered by the CFHT images is approximately 2.2 on a side and is slightly offset from the cluster center, although the center is in the field.

We also made use of several HST datasets. $B V$ data were taken from the public online data archive of Piotto et al. (2002). Additional unreduced images in the ultraviolet were taken from the HST archives (proposal ID: 6607, P.I. Ferraro.). The UV images were reduced using version 1.1.5b of the HSTPHOT package (Dolphin 2000a), which is optimized for the reduction of WFPC2 images. The images were preprocessed using the mask, getsky, crmask, and hotpixel routines to eliminate saturated, vignetted, and hot pixels as well as those with cosmic ray hits. If the images in a set were dithered, initial guesses for the pixel offsets relative to a reference frame were taken from WFPC2 Associations webpage ${ }^{1}$ in order to align the images before cosmic ray rejection. The hstphot routine was then used to conduct PSF photometry using precomputed PSFs ${ }^{2}$. We used flight-system magnitudes in subsequent analysis of this dataset. Positions derived from HST images were transformed to right ascension and declination using the IRAF task metric. These coordinates were used as the basis for the positions [relative to the cluster center given by Harris 1996:

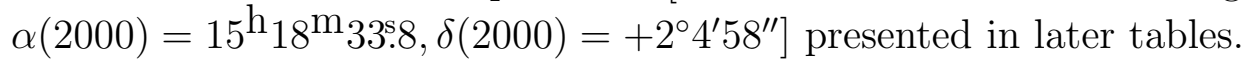

\footnotetext{
${ }^{1}$ http://archive.eso.org/archive/hst/wfpc2_asn

${ }^{2}$ http://purcell.as.arizona.edu/hstphot/
} 


\section{The BSS sample}

We attempted to obtain a reliable global BSS sample by merging datasets for the core of the cluster (two HST datasets and the CFHT dataset) with $r \lesssim 150^{\prime \prime}$, and the CTIO dataset for the outskirts of the cluster $\left(r>150^{\prime \prime}\right)$. See Table 2 for an example of the photometry for the candidate BSSs.

Because there is not a reliable and uniform set of photometry covering all of the stars in the cluster, by necessity we had to pay careful attention to our selection criteria so that the samples in different parts of the cluster are drawn from consistent portions of the CMD. In this section we focus on the selection of a reliable set of bright blue stragglers separately for the cluster core and outskirts.

\subsection{The Core Sample}

Photometric data for the stars in the cluster core were matched by sky position. Figure 1 shows the regions covered by the three datasets. Because these fields did not completely overlap and different filter combinations were used for each field, the brightness and color information available varied from star to star. We used selection criteria similar to those used by Ferraro et al. (1997b) in their study of M3. BSSs were selected from the four CMDs shown in Fig. 2. The $(V, B-V)$ and $\left(m_{255}, m_{255}-m_{336}\right)$ HST CMDs were the primary choice for BSS selection due to the high spatial resolution. We defined the most reliable "bright" BSS (BBSS) sample as stars with $B<17.81$ or $m_{255}<18.35$ (see $\S 4$ for the motivation behind these cuts). The $\left(m_{555}, m_{336}-m_{555}\right)$ CMD was also used in the selection, but mainly as a secondary check for portions of the field that did not overlap with the $(V, B-V)$ CMD. We identified a star as a BSS if it was selected in both the $(V, B-V)$ and $\left(m_{255}, m_{255}-m_{336}\right)$ CMDs if the star was covered by both HST fields, or in just one if in a part of the field where the HST observations did not overlap.

Reliable faint BSSs (FBSSs) were selected differently for each CMD. For the $(V, B-V)$ CMD, FBSSs were selected if they were significantly bluer $(B-V=0.37)$ and brighter ( $V=18.57$; Sandquist et al. 1996) than the MSTO and below the BBSS cutoff. The color cut was chosen to avoid photometric scatter near the turnoff. FBSSs were selected in the $\left(m_{255}, m_{255}-m_{336}\right)$ CMD by choosing all of the stars brighter than the majority of the photometric scatter $\left(m_{255}<18.9\right)$ and below the BBSS cutoff, though this is likely to be contaminated by optical blends to some degree. Stragglers in the $\left(m_{555}, m_{336}-m_{555}\right)$ CMD were selected if they were brighter than the MSTO and outside of the scatter. A faint limit cut of $m_{555}<18.31$ was imposed. We emphasize, however, that this faint sample was 
not used in the main comparisons later in the paper because it is prone to contamination resulting from stars with large photometric errors and optically-blended stars.

Bright or faint BSSs were only selected from the CFHT data $(B, B-I)$ if no data were available from HST photometry because blending effects were noticeable for stars nearest the cluster center. For the $(B, B-I)$ CMD, FBSSs were selected if they were bluer $(B-I=1.0)$ and brighter ( $B=19.04$; Sandquist et al. 1996) than the MSTO and below the BBSS cutoff. The candidate FBSSs closest to the MSTO appeared to be within the scatter and were not included, although FBSSs could exist in that region. Once again, we emphasize our purpose in tabulating FBSSs is simply to identify candidates for future work - we have not included them in the main analysis in this paper.

Stars that were not selected as BSSs that appear in the CMDs in regions that BSSs occupy were eliminated based on information from other overlapping photometric datasets. In total, 48 bright BSSs and and 47 faint BSSs were selected.

\subsection{The Outer Region Sample}

The outer region of M5 consists of two ground-based data sets from CTIO: one in $B$ and $V$ and the other in $B$ and $I$. Stars were again matched by position, and put on a common coordinate system with the core stars. The regions covered by the HST and CFHT core fields were then removed from consideration. Due to photometric scatter in the groundbased data, we only selected stars that were above the BBSS magnitude cutoff $(B<17.81)$ used in the core sample. The bright BSSs were then selected in the same manner as the core sample. A star was considered a BBSS if $B<17.81$ and $B-I<1.05$.

As before, stars that appear in the $(V, B-V)$ CMD that were not selected as BBSSs were rejected based upon their positions in the $(B, B-I)$ CMD. The $B$ and $I$ images were taken under better seeing conditions than the $V$ images, and had fewer issues with blended star images. Based on these selections, we identified 11 BBSSs outside of our core sample.

Faint BSSs $(B>17.81)$ were selected after an additional radial cut was implemented. Stars just outside the HST and CFHT fields were still subject to significant crowding effects and to minimize these effects a radial cut $\left(r>152^{\prime \prime}\right)$ was implemented. The selection criteria are discussed in more detail in $\S 4$. Only the $(B, B-I)$ CMD was used in the selection of faint candidate BSSs due to the noticable blending effects seen in the $(V, B-V)$ CMD even with the radius cut. A total of 13 FBSSs were selected. The selected BBSSs and FBSSs are shown in Fig. 3. Fig. 4 shows the cluster position of the BBSSs from the core and outer region samples. We emphasize again that the FBSSs were not used in the analysis below, 
and are included for completeness.

\section{Comparisons with Other Studies}

To make fair comparisons with studies of stragglers in other clusters, we have taken the BSSs derived from all the photometry we had available (as described above) and applied the photometric cuts described in those studies. We have also applied the same photometric cuts to samples from single CMDs to try to choose BSSs in a manner most accurately matching that of the original paper. In this way, we can minimize systematic errors and gauge possible pitfalls involved in selecting stragglers using information from a single CMD.

\subsection{Comparison with Ferraro et al. (1997b)}

Ferraro et al. (1997b) examined M3 using multiple wavelengths [filters F255W, F336W, F555W $(V)$, and F814W $(I)$ in the core, and $B, V$, and $I$ in the outskirts] in order to obtain a global BSS sample for the cluster. They used a cut in $m_{255}$ to select their core BSS sample, and a cut in $B$ to select their outer sample. Ferraro et al. used the $m_{255}-m_{336}$ color to select their core BSS sample because the area of the CMD occupied by BSSs is very distinct from the locuses of single stars in other evolutionary phases. They divided their sample into two sub-samples: BBSSs and FBSSs. BBSSs were selected in M3 if $m_{255}>19$.0, while FBSSs had $19.0<m_{255}<19.4$ (though FBSSs were not used in their analysis). The BSS sample was split in order to avoid contamination arising from stars with large photmetric error or optical blends. Later in this paper, we will compare the radial distribution of BBSSs obtained for M3 by Ferraro et al. (1997b) with the BBSS radial distribution from M5. Here we first examine the consistency of these cuts because we have HST photometry in $m_{255}$ and $B$ (more precisely, F439W calibrated to $B$ ) in a portion of the core field.

To reliably compare our core sample with that of M3, we needed to determine BBSS sample cuts that were compatible with those used by Ferraro et al. (1997b). To do this, we made our photometry cuts at the same position relative to the cluster turnoff. M3 has a MSTO magnitude of $B=19.54$ (Ferraro et al. 1997a) and Ferraro et al. (1997b) used a faint cut for the BBSS sample at $B=18.6$. We used this difference of 0.94 mag to define the faint end of the sample, using the MSTO value for M5 ( $B=19.04$; Sandquist et al. 1996) to define the cut at $B=18.1$. In order to select BBSSs in the $\left(m_{255}, m_{255}-m_{336}\right)$ CMD we followed the method used in (Ferraro et al. 2003) (see $\S 4.2$ for details). BBSSs were not directly chosen from the $\left(m_{555}, m_{336}-m_{555}\right)$ CMD, which covered the same field, although 
it was used as a check.

Because some stars were observed in both HST pointings, we were able to check the consistency of the two faint cuts. We found that ten stars fell below the selection line in the $\left(m_{255}, m_{255}-m_{336}\right)$ CMD though they satisfied the requirements for a BBSS in the $(V, B-V)$ CMD (see the right panels of Fig. 5). Two stars that were selected based on the $\left(m_{255}, m_{255}-m_{336}\right)$ CMD would not have been selected in the $(V, B-V)$ because they were redder than the turnoff. Nevertheless, if we keep all stars that were selected using either the $B$ or $m_{255}$ cuts following Ferraro et al. (1997b) and Ferraro et al. (2003), we end up with 59 BBSSs from the core sample.

The inconsistency in the samples derived using cuts in different filters indicates that the faint cut needs to be adjusted for one of the filters. In order to have as consistent a sample as possible, we shifted the BBSS cut brightward in $B$. A BBSS cut of $B=17.81$ improves the agreement between the samples in $(V, B-V)$ and $\left(m_{255}, m_{255}-m_{336}\right)$. The one star below the BBSS cut line in the $\left(m_{255}, m_{255}-m_{336}\right)$ CMD was kept in because of its position among the other BBSSs in the $\left(m_{555}, m_{336}-m_{555}\right)$ CMD. The two stars that fell just below the BBSS cut line in the $(V, B-V)$ CMD were kept in due to their positions in the $\left(m_{255}, m_{255}-m_{336}\right)$ CMD. This choice gives us a sample of 48 BBSSs from the core of M5, as shown in the left panels of Fig. 5.

The region outside of the core samples was scrutinized in the same fashion. Using our BBSS cut of $B=17.81$, we identified 11 BBSSs. (Once again, only the BBSSs were used for comparisons.) If we instead use a fainter cut in $B$ consistent with that of Ferraro et al. (1997b), we obtain 23 BBSSs.

\subsection{Comparison with Ferraro et al. (2003)}

In another study by Ferraro et al. (2003), BSSs were identified in the cores of five globular clusters using HST data in the $F 255 W$ and $F 336 W$ filters, and were compared to results for M3 (Ferraro et al. 1997b) (see §4.1). Photometry of M5 was taken as part of the same program (program ID 6607, PI: Ferraro), but has not been presented in the literature.

In order to make the comparison, we shifted our $\left(m_{255}, m_{255}-m_{336}\right)$ CMD in magnitude and color to overlie the brightest HB stars in the M3 CMD, following the method used by Ferraro et al. (2003). The shift in the ultraviolet wavelength F255W was done by lining up the brightest portion of the HB of M5 with that of M3. The straight line cut at magnitude $m_{255}=19.0$ in F255W for M3 was then used as the dividing line for our BBSS sample. This magnitude cut falls at $m_{255}=18.35$ for M5. 
Using our best BSS sample (selected using information from other CMDs when available), we obtain a count of 31 BBSSs. Fig. 2 shows the 31 BBSSs obtained from our BSS list. The one star below the BBSS cutoff was kept because of its placement in the $\left(m_{555}, m_{336}-m_{555}\right)$ CMD. If we use only information from the $\left(m_{255}, m_{255}-m_{336}\right)$ CMD to select stars, the sample size is 34 BBSSs.

The filled black circles in figure 2 are the extra BBSSs that would have been selected if we had used only the information from the $\left(m_{255}, m_{255}-m_{336}\right)$ CMD. In other CMDs, these stars were found far outside the regions where BSSs were found. The bluest of these stars in the $\left(m_{255}, m_{255}-m_{336}\right) \mathrm{CMD}$ of figure 2 was not found in the $(V, B-V)$ CMD, but had a $m_{336}-m_{555}$ color of -1.862 . The next bluest star had a $V$ magnitude of 18.4963 and a $B-V$ color of 1.4198 , and fell just above the SGB in the $\left(m_{555}, m_{336}-m_{555}\right)$ CMD. The next star (moving redward in $m_{255}-m_{336}$ ) did not appear in the $(V, B-V)$ CMD, but had $m_{336}-m_{555}=0.993$, which put it redward of the RGB in the $\left(m_{555}, m_{336}-m_{555}\right)$ CMD. The reddest of the four stars in $m_{255}-m_{336}$ fell within the RGB in the $(V, B-V)$ CMD and just blueward of the RGB in the $\left(m_{555}, m_{336}-m_{555}\right)$ CMD.

\subsection{Comparison with Piotto et al. (2004)}

Piotto et al. selected blue stragglers in the cores of 56 globular clusters (including M5) using $B V$ data from HST. We used the same images (program ID 8118, PI: Piotto) in selecting a portion of our core sample for comparison. Piotto et al. selected stragglers brighter than the MSTO of each cluster, and for M5, the MSTO has a $V=18.57$ and $B-V=0.47$ (Sandquist et al. 1996). We chose all BSS (selected with information from other CMDs when available) with $V<18.57$ and $B-V<0.37$. Our color cut was chosen to avoid photometric scatter near the main sequence. The sample of 40 stars for this comparison can be seen in Fig. 6. Again, stars within this region of the CMD that were not circled were rejected using information from other CMDs. Using the raw $(V, B-V)$ CMD and the above selection boundaries, we obtain a sample of 55 stars.

\section{HB Stars and the Brightest Stragglers}

To examine the frequency of stragglers, we used the HB and RR Lyrae stars tabulated previously by Sandquist et al. (2004) as a comparison population. Six stars in our core sample of BSS were identified as probable blends of HB and RGB stars by Sandquist et al. based on their position redder than the blue tail of the HB. (These stars had HB identification 
numbers of $107,201,221,250,268$, and 276.) Using ultraviolet data from HST (with its better temperature sensitivity for the hottest HB stars), we find that they are significantly fainter than the HB locus, which makes us confident that they are bright BSS, and not blends of HB stars. One star in our outer region sample (HB ID number 302) was also reclassified as a BSS based upon its position redder than the blue tail of the HB in both of the ground-based CMDs. No known RR Lyrae stars made it into our BSS selections.

The BSSs that were mistakenly identified as HB stars by Sandquist et al. (2004) are an interesting class of BBSSs. The most likely formation mechanism for these stars is stellar collisions for the simple reason that a merger of a close binary star or mass transfer within a binary cannot produce a remnant that is massive and luminous enough. The merger of at least three stars (resulting from a collision involving a binary and at least one other star) seems to be the only feasible means of accomplishing this.

Finally, we have three stars to add to the list of HB stars in Sandquist et al. (2004) (see Table 3). Star 1 has a proper motion estimate (Rees 1993) and was identified as a likely non-member; however, it was the faintest star in the study and its membership probability is quite uncertain. It falls near the middle of the blue tail of the HB in our ground-based $B V$ dataset, and so we consider it likely to be an HB star. Star 2 was mistakenly excluded by Sandquist et al. (2004) due to a bad $V$ band measurement that prevented it from being identified as an HB star. However, based upon its position in both of our CTIO CMDs near the tip of the blue tail of the HB, it should be included. Star 3 is close to a bright giant star and its photometry may have been contaminated. Its CMD position in the $B V$ HST data (brighter and bluer than the HB) leads us to believe it is an evolved HB star.

\section{Results}

\subsection{The BBSS Radial Distribution}

In most clusters in which stragglers have been surveyed, the stragglers have been found to be more centrally concentrated than comparison populations (such as HB or RGB stars). For example, Ferraro et al. (1997b) showed that the BBSSs of M3 were more centrally concentrated than the cluster's RGB stars. In Fig. 7, we compare the cumulative radial distributions of the 59 total $\mathrm{BBSSs}^{3}$ and the $513 \mathrm{HB}$ stars in the same field (Sandquist et al. 2004). The figure shows that the BBSSs are more centrally concentrated than the HB stars,

\footnotetext{
${ }^{3}$ This sample is composed of our samples of 48 core stragglers and 11 envelope stragglers using the most consistent choice of faint cuts, as described in $\S 4.1$.
} 
and a Kolmogorov-Smirnov (K-S) test gives a probability of 0.0015 that the two samples come from the same distribution.

For the globular cluster M3, Ferraro et al. discovered a bimodal distribution with a centrally-concentrated core population, a sparsely-populated "zone of avoidance", and an outer population. Sigurdsson et al. (1994) attempted to explain the origin of the separate BSS populations, suggesting that all BSSs are formed via collisions involving primordial binaries in the cluster core. The BSS that forms can be kicked into the outskirts of the cluster if the "spectator" star (in binary-single star collision) is ejected from the system or if there is sufficient mass loss during the collision to perturb the center of mass. Sigurdsson et al. 1994 also suggest that BSSs formed or kicked out to $\lesssim 7 r_{c}$ will quickly migrate back into the core via dynamical friction, while those kicked out beyond $\sim 7 r_{c}$ are effectively "parked" in the cluster outskirts. The two different timescales will naturally give rise to the "zone of avoidance" seen in M3.

Since the study by Ferraro et al. (1993), similar distributions have been confirmed in a few other massive clusters (M55, Zaggia et al. 1997; 47 Tuc, Ferraro et al. 2004; NGC 6752, Sabbi et al. 2004), while a unimodal distribution was observed in one low-mass cluster (Palomar 13, Clark et al. 2004). Because M5 has a structure that is quite similar to that of M3, we can test whether structurally-similar clusters produce similar straggler distributions. According to the values in Table 1 (Harris 1996; McLaughlin \& van der Marel 2005), M3 and M5 have similar concentrations $c$, while M5 has a $17 \%$ smaller core radius $r_{c}$ than M3, and slightly smaller half-light radius $r_{h}{ }^{4}$.

In Fig. 8, we compare the radial distribution of our BBSS sample with that of Ferraro et al. (1997b) for M3 when scaled horizontally for differences in core radius. The quantity $R_{B S S}$ (Ferraro et al. 1993) is

$$
R_{B S S}=\frac{\left(N_{B S S} / N_{B S S}^{t o t}\right)}{\left(L^{\text {sample }} / L_{\text {tot }}^{\text {sample }}\right)} .
$$

The radial distribution for M5, like that for M3, has a bimodal nature, although our field was not large enough to identify where the outer population of BSS peaks. Scaling by core radius is a natural choice if the distribution is modified by mass segregation of stragglers through

\footnotetext{
${ }^{4}$ The half-light radius is more difficult to determine than core radius because it depends on accurate knowledge of the whole surface brightness profile. We have quoted values from the Wilson models presented in McLaughlin \& van der Marel (2005) because they follow the distribution at large distance from the cluster center more closely than King models do. Based on this, the half-light radius of M5 is about $11 \%$ smaller than that of M3. When King models are used instead, M3's half light radius becomes considerably larger, so that M5 appears to have a $45 \%$ smaller half-light radius.
} 
dynamical interactions with other cluster stars. This scaling does indeed cause the distributions to overlie each other in the central regions of the clusters. Nonetheless, we examined alternate ways of scaling the radial distributions to compensate for the small structural differences between clusters. Scaling by half-light radius introduces more uncertainty because the half-light radii are harder to determine (see the footnote in \$6.1). In the case of M3 and M5 though, scaling by half-light radius would be very similar to scaling by core radius. We also tried using $L^{\text {sample }} / L_{\text {tot }}$ (derived from King models) as a horizontal coordinate, although this again did not produce an alignment of the straggler distributions. Sabbi et al. (2004) provide additional evidence for a scaling with core radius in their examination of straggler populations in the cores of 8 clusters from HST data, finding that the half-sample radii fell at approximately $1 r_{c}$. (Their samples, however, only included stars within single HST WFPC2 fields of view.)

When the two cluster distributions are scaled with core radius, we find that they agree to within $2 \sigma$ out to $r / r_{c} \lesssim 13$. M5 appears to have a wider "zone of avoidance" $\left(4 \lesssim r / r_{c} \lesssim 14\right)$ than M3 ( $\left.4 \lesssim r / r_{c} \lesssim 8\right)$, and a lower frequency of stragglers there. Both of these facts imply that M5's structure is somewhat more dynamically evolved (stars that started farther from M5's center having been dynamically relaxed to the core).

BSS radial distributions have only been produced for a handful of other clusters. Figure 9 shows the scaled radial distributions for M5, M3 (Ferraro et al. 1997b), 47 Tuc, and NGC 6752. (The distribution for NGC 6752 uses the quantity $\left(N_{B S S} / N_{B S S}^{t o t}\right) /\left(N_{H B} / N_{H B}^{t o t}\right)$ as a stand-in for $R_{B S S}$.) All four clusters have a core BSS sample that falls within about $4 r_{c}$ of the center. The minima of the distributions all fall in the range $5 \lesssim r / r_{c} \lesssim 12$.

Mapelli et al. (2004) created dynamical models to understand the BSS distributions in 47 Tuc, finding it necessary to employ two separate populations of BSSs: a core population and an outer population, compared to a single core population described by Sigurdsson et al. (1994). The region $r<20 r_{c}$ (containing the core and current zone of avoidance) produced BSSs primarily through stellar collisions, which were concentrated to the center of the cluster through the action of dynamical friction. The region beyond $20 r_{c}$ was populated with BSSs born in primordial binary systems via mass transfer, and remaining in the cluster outskirts due to the weakness of dynamical friction in the low density environment. These ingredients can naturally produce zones of avoidance seen in all four clusters in Fig. 9, although differences in cluster history and current cluster characteristics could leave signatures on the straggler distribution.

Mapelli et al. used the timescale for dynamical friction (Binney \& Tremaine 1987)

$$
t_{D F}=\frac{3}{4 \ln (\Lambda) G^{2}(2 \pi)^{1 / 2}} \frac{\sigma^{3}}{M \rho(r)}
$$


to predict the radius at which the zone of avoidance should occur, assuming circular star orbits within the cluster, cluster ages of about $12 \mathrm{Gyr}$, typical BSS mass $M$ of about $1.5 M_{\odot}$, Coulomb logarithm $\ln \Lambda \approx 10$, and appropriate King models for the structure. Using velocity dispersion values $\sigma$ given by Pryor \& Meylan (1993), we obtained expected $r / r_{c}$ values for M5 ( 8.1), M3 $(\sim 6.3), 47$ Tuc $(\sim 8.3)$, and NGC $6752(\sim 14.6)$ that agree nicely with the distributions shown in Fig. 9. The assumption of circular orbits is probably the most questionable - if the straggler orbits are significantly eccentric, this would shorten the dynamical friction timescale since the stragglers would periodically sample higher stellar densities and higher rates of dynamical friction.

In Fig. 10 we plot the cumulative radial distributions of the BBSSs of M5 (dashed line) and M3 (solid line). A K-S test applied to the two lines gives a $40 \%$ probablility that the two radial distributions are derived from the same underlying distribution. The inconclusive nature of the test results mostly from the relatively small number of bright stragglers in M5 and the fact that the largest differences are in the sparsely populated outskirts of the clusters. Because K-S tests are not as sensitive to differences in the tails of distributions, we conducted Monte Carlo simulations by randomly drawing 58 BBSSs (the size of the M5 sample which spans the M3 sample, $r<360^{\prime \prime}$ ) from the M3 sample (114 stars). We looked for trials in which the maximum deviation between the cumulative radial distributions was greater than 0.14 (the measured deviation between the M5 and M3 samples), with the smaller sample having the larger cumulative distribution value. For ten million trials, we found that the frequency of this was $9.5 \%$, which indicates that the M5 distribution is marginally inconsistent with the M3 distribution.

The largest differences between the BBSS populations of M5 and M3 are in the "zone of avoidance". In making these comparisons, we must keep in mind that the ordinates for the two distributions are not identical, and that these tests do not account for the possibility of systematic errors in the independent variable. For example, errors in the core radii would affect the comparison of the distributions. In this paper, we used $r_{c}=25^{\prime \prime}$ for M3 from Webbink (1985) as used in Ferraro et al. (1993) and subsequent papers. This value differs slightly from some more recent fits to surface brightness data (22'.9, McLaughlin \& van der Marel 2005; 30'2, Trager et al. 1995). By comparison, the most recent determinations for M5 agree very well (26"4 , Lehmann \& Scholz 1997; 26"3, McLaughlin \& van der Marel 2005; 24", Trager et al. 1995). Using the most recent value for M3 from the King model fit of McLaughlin \& van der Marel (which agree well with values that can be derived by interpolations in the surface brightness data), the differences between the M3 and M5 distributions become only slightly more significant (a K-S test returns a $31 \%$ probability that the M3 and M5 distributions come from the same underlying distribution, and the Monte Carlo simulations indicate that stars drawn from the M3 distribution produce M5-like distributions only 6.7\% 
of the time). More definitive conclusions will require larger straggler samples.

Regardless, King models scaled by core radius and by central density agree very well for $r / r_{c} \lesssim 10$ for a wide range of concentrations $c$ (for example, Fig. 4-9 of Binney \& Tremaine 1987). So if populations of stars in two different clusters follow the underlying light distributions (described by an appropriate King model), scaling by the core radius

should minimize differences in the scaled distributions. The agreement of the two blue straggler distributions for $r / r_{c}<2$ provides some supporting evidence for this choice.

\subsection{The Total BSS Population}

Apart from the radial distributions, the sizes of straggler populations vary from cluster to cluster. Piotto et al. (2004) found that the relative frequency of stragglers, defined as

$$
F_{B S S}=\frac{N_{B S S}}{N_{H B}}
$$

is significantly anti-correlated with total cluster luminosity. However, the scatter in the anticorrelation discussed by Piotto et al. 2004 is evidence of some additional variation from cluster to cluster. Using the comparison sample obtained from our total BSS list for M5 (stragglers selected using all available CMD information), we find $F_{B S S}=0.239 \pm 0.042$. If we select BSSs in a manner mimicking that of Piotto et al. (2004), we find $F_{B S S}=0.329 \pm 0.051$. Both of these values are similar to the M5 value $(\sim 0.26)$ used in Fig. 1 of Piotto et al. (2004). Regardless of the exact value, M5 (and M3) fall near the upper envelope of clusters in the trend of $\log F_{B S S}$ with $M_{V_{t}}$.

We then examined the global sample of bright stragglers for M3 and M5. The global sample for M3 found by Ferraro et al. (1997b) consisted of 114 BBSSs with $r<360^{\prime \prime}$. According to a King model for the cluster, this radius corresponds to a fractional contained luminosity of 0.697 . To compare the global sample for M3 to that of M5, we used a global sample selected with the same criteria used by Ferraro et al. (1997b) out to a radius having the same fractional contained luminosity $\left(r<244^{\prime \prime}\right)$. As described earlier, we have also made a strong effort to ensure that our photometric selection criteria matched the ones used for M3. Our sample contains $82 \mathrm{BBSSs}$, returning a frequency $F_{B S S}=0.159 \pm 0.019$ for M5, compared to $0.197 \pm 0.020$ for M3. Though M5 is the more luminous cluster, it contains about $20 \%$ fewer bright stragglers than M3.

Using a single HST WFPC2 field of view centered on the cores of 8 clusters, Sabbi et 
al. (2004) compared the specific frequencies of bright BSS defined as

$$
F_{H B}^{B B S S}=\frac{N_{B B S S}}{N_{H B}} .
$$

Using the 31 BBSSs found in our UV sample, we obtain a frequency of $0.19 \pm 0.04$ for M5. When the BBSSs are selected using the method of Ferraro et al. (1997b), we find $F_{H B}^{B B S S}=0.21 \pm 0.04$. These two values for M5 are statistically consistent and give M5 one of the lowest values of the clusters listed in Table 3 of Sabbi et al. (2004). Sabbi et al. found that NGC 6752 had a low specific frequency $(0.18 \pm 0.04)$ of bright stragglers, while Ferraro et al. (1997b) found a frequency of $0.28 \pm 0.04$ for M3. The specific frequencies for M5 and M3 do not differ greatly, as was the case for the radial distributions. This again shows that there is little difference between the two clusters' BSS populations. We must keep in mind that even among the clusters examined by Sabbi et al. there was a wide range of cluster luminosities represented, so that simple comparisons of specific frequencies could be misleading.

If the brightest blue stragglers are more likely to be produced by collisional processes (mergers of two or more stars), it is worth asking whether the bright straggler frequencies show the same anti-correlation with cluster luminosity that the larger samples of Piotto et al. do. As Piotto et al. showed, there appears to be a change in the luminosity function of stragglers between the brightest $\left(M_{V_{t}} \lesssim-8.8\right)$ and faintest $\left(-7 \gtrsim M_{V_{t}} \gtrsim-8.8\right)$ clusters in their sample. A least-squares fit to the $\log F_{H B}^{B B S S}$ values $\left(M_{V_{t}}\right.$ obtained from Harris 1996) (see Fig. 11) for M5 and the 8 clusters studied by Sabbi et al. (2004) produces a slope (0.28) that is significant at the $2.5 \sigma$ level. This slope is similar to the average slope seen in Fig. 2 of Sandquist (2005) for total samples in clusters with $-4 \gtrsim M_{V_{t}} \gtrsim-10$. So, with the limited (in number of clusters and in luminosity range) cluster sample available at present, the bright stragglers appear to be behaving similarly to the total samples, although if we remove the lowest luminosity cluster (NGC 288) from the list, the fitted slope is only $0.9 \sigma$ different from zero.

The relative BBSS population sizes of M5 and M3 are very similar, as one might expect for two clusters having similar dynamical properties. Their frequency values differ slightly, possibly in part due to the small differences in their total cluster luminosities. The two clusters may differ in the distribution of their stragglers. M5's "zone of avoidance" appears to be wider than that of M3 and there is a larger percentage of the stragglers in this zone in M3 than in M5. Both of these facts are consistent with the idea that M5's stragglers are more dynamically relaxed than those of M3, separating the core and envelope stragglers to a greater degree. 


\section{Conclusions}

As was described years ago by Ferraro et al. (1997b), multi-wavelength photometry is truly necessary to reliably identify BSSs by utilizing cross-checks between the different bands. The BSSs mistakenly identified as HB stars by Sandquist et al. (2004) are a prime example of this. For clusters with blue HB morphologies, the identification of bright blue stragglers is not a trivial matter. These BSSs are of special interest because the brightest ones are likely to be binary star collision products. The UV bands are of great importance when picking out these BSSs because they can most cleanly separate stragglers from the hotter HB stars. Optical colors are simply not color-sensitive enough for the hottest stars.

Our results from the comparisons of the BSS populations confirm that M5 and M3 have similar dynamical characteristics. Both of their radial distributions show bimodality, implying distinct core and envelope populations. When scaled for differences in core radius, the two distributions largely overlie each other - the agreement is best in the core, though M5 appears to have a larger "zone of avoidance" and a secondary frequency peak that is farther from the cluster center. The BSS frequencies for the two clusters are quite similar as well. The slightly smaller frequency values for M5 are consistent with M5's higher total luminosity when considering the anti-correlation between frequency and cluster luminosity mapped out by Piotto et al. (2004).

While cluster luminosity seems to have been established as a primary predictor of straggler frequency, there does appear to be significant scatter that results from another source. Future work to understand this scatter should include an investigation into other clusters with luminosities comparable to M3 and M5, such as M2, M13, M53, and NGC 5824. In the clusters examined by Piotto et al., there is approximately a factor of 3 difference in frequencies among clusters with integrated luminosities similar to M5. Since these clusters have large total straggler populations, it would also be possible to examine the radial distibutions in maximum detail.

We would like to thank undergraduate Tom Jackson for his contributions to a preliminary study of M5's stragglers. Jackson was a participant in the NASA College Summer Research Program at Northwestern University. This work has been funded through grants AST 00-98696 and 05-07785 from the National Science Foundation to E.L.S. and M.B.

\section{REFERENCES}

Binney, J., \& Tremaine, S. 1987, Princeton, NJ, Princeton University Press, 1987, 747 
Clark, L. L., Sandquist, E. L., \& Bolte, M. 2004, AJ, 128, 3019

Dolphin, A. E. 2000a, PASP, 112, 1383

Ferraro, F. R., Pecci, F. F., Cacciari, C., Corsi, C., Buonanno, R., Fahlman, G. G., \& Richer, H. B. 1993, AJ, 106, 2324

Ferraro, F. R., Carretta, E., Corsi, C. E., Fusi Pecci, F., Cacciari, C., Buonanno, R., Paltrinieri, B., \& Hamilton, D. 1997, A\&A, 320, 757

Ferraro, F. R., Paltrinieri, B., Fusi Pecci, F., Cacciari, C., Dorman, B., Rood, R. T., Buonanno, R., Corsi, C. E., Burgarella, D., \& Laget, M. 1997, A\&A, 324, 915

Ferraro, F. R., Sills, A., Rood, R., Paltrinieri, B., \& Buonanno, R. 2003, ApJ, 588, 464

Ferraro, F. R., Beccari, G., Rood, R. T., Bellazzini, M., Sills, A., \& Sabbi, E. 2004, ApJ, 603,127

Harris, W.E. 1996, AJ, 112, 1487

Lehmann, I., \& Scholz, R.-D. 1997, A\&A, 320, 776

Mapelli, M., Sigurdsson, S., Colpi, M., Ferraro, F. R., Possenti, A., Rood, R. T., Sills, A., \& Beccari, G. 2004, ApJ, 605, L29

McLaughlin, D. E., \& van der Marel, R. P. 2005, ApJS, 161, 304

Piotto, G., et al. 2002, A\&A, 391, 945

Piotto, G., et al. 2004, ApJ, 604, L109

Pryor, C., \& Meylan, G. 1993, ASP Conf. Ser. 50: Structure and Dynamics of Globular Clusters, 50, 357

Rees, R. F. 1993, AJ, 106, 1524

Sabbi, E., Ferraro, F. R., Sills, A., \& Rood, R. T. 2004, ApJ, 617, 1296

Sandage, A. R. 1953, AJ, 58, 61

Sandquist, E. L., Bolte, M., Stetson, P. B., \& Hesser, J. E. 1996, ApJ, 470, 910

Sandquist, E. L. \& Bolte, M. 2004, ApJ, 611, 323

Sandquist, E. L. 2005, ApJ, 635, 73 
Sigurdsson, S., Davies, M. B., \& Bolte, M. 1994, ApJ, 431, L115

Trager, S. C., King, I. R., \& Djorgovski, S. 1995, AJ, 109, 218

Webbink, R. F. 1985, in IAU Symp. 113, Dynamics of Star Clusters, eds. J. Goodman \& P. Hut (Dordrecht: Reidel), 541

Zaggia, S. R., Piotto, G., \& Capaccioli, M. 1997, A\&A, 327, 1004

This preprint was prepared with the AAS LATEX macros v5.2. 
Table 1. Approximate Structural Parameters of M3 and M5

\begin{tabular}{lcc}
\hline \hline & $\mathrm{M} 3$ & $\mathrm{M} 5$ \\
\hline$M_{V_{t}}{ }^{\mathrm{a}}$ & -8.93 & -8.81 \\
$\log j_{0}\left(L_{\odot} \mathrm{pc}^{-3}\right)^{\mathrm{b}}$ & 3.47 & 3.90 \\
$c^{\mathrm{b}}$ & 1.89 & 1.71 \\
$r_{c}(\operatorname{arcsec})^{\mathrm{b}}$ & $22^{\prime \prime} .9$ & $26^{\prime \prime} .3$ \\
$r_{c}(\mathrm{pc})$ & 1.2 & 0.96 \\
$r_{h}(\mathrm{pc})^{\mathrm{b}}$ & 3.7 & 3.3 \\
$d(\mathrm{kpc})^{\mathrm{a} b}$ & 10.4 & 7.5 \\
\hline
\end{tabular}

${ }^{a}$ Harris (1996), updated February 2003

${ }^{\mathrm{b}}$ McLaughlin \& van der Marel (2005) 
Table 2: Candidate BSSs. The top half are core BSSs and the bottom half are BSSs from the outer region sample.

\begin{tabular}{crrrrrrc}
\hline \hline Star & $\Delta \alpha(\operatorname{arcsec})^{\mathrm{a}}$ & $\Delta \delta$ (arcsec $^{\mathrm{a}}$ & Filter $^{\mathrm{b}}$ & Magnitude & Filter $^{\mathrm{b}}$ & Magnitude & Telescope \\
\hline 1 & 0.689 & 25.780 & $\mathrm{~V}$ & 15.5975 & $\mathrm{~B}$ & 15.9888 & HST \\
1 & 0.689 & 25.780 & $\mathrm{~B}$ & 16.0518 & $\mathrm{I}$ & 15.0094 & CFHT \\
1 & 0.689 & 25.780 & $\mathrm{~F} 5$ & 15.7100 & $\mathrm{~F} 3$ & 15.9610 & HST \\
& & & $\mathrm{F} 2$ & 16.8150 & & & \\
2 & 1.534 & -2.248 & $\mathrm{~V}$ & 17.3030 & $\mathrm{~B}$ & 17.5303 & $\mathrm{HST}$ \\
2 & 1.534 & -2.248 & $\mathrm{~F} 5$ & 17.2750 & $\mathrm{~F} 3$ & 17.4190 & HST \\
& & & $\mathrm{F} 2$ & 17.7770 & & & \\
3 & 1.647 & 18.375 & $\mathrm{~V}$ & 18.1037 & $\mathrm{~B}$ & 18.3390 & HST \\
3 & 1.647 & 18.375 & $\mathrm{~B}$ & 18.2611 & $\mathrm{I}$ & 17.3427 & CFHT \\
3 & 1.647 & 18.375 & $\mathrm{~F} 5$ & 18.1090 & $\mathrm{~F} 3$ & 18.0140 & HST \\
& & & $\mathrm{F} 2$ & 18.7390 & & & \\
4 & 1.690 & -2.458 & $\mathrm{~B}$ & 17.9223 & $\mathrm{I}$ & 16.9373 & CFHT \\
\hline \hline 1 & 94.010 & -378.603 & $\mathrm{~V}$ & 17.0280 & $\mathrm{~B}$ & 17.3258 & CTIO \\
1 & 94.010 & -378.603 & $\mathrm{~B}$ & 17.3298 & $\mathrm{I}$ & 16.7495 & CTIO \\
2 & -112.995 & 307.413 & $\mathrm{~V}$ & 16.7213 & $\mathrm{~B}$ & 16.9203 & CTIO \\
2 & -112.995 & 307.413 & $\mathrm{~B}$ & 16.9223 & $\mathrm{I}$ & 16.5535 & CTIO \\
3 & -163.805 & -47.989 & $\mathrm{~V}$ & 17.1095 & $\mathrm{~B}$ & 17.5135 & CTIO \\
3 & -163.805 & -47.989 & $\mathrm{~B}$ & 17.5144 & $\mathrm{I}$ & 16.5490 & CTIO \\
4 & -165.143 & -129.545 & $\mathrm{~V}$ & 17.4614 & $\mathrm{~B}$ & 17.7707 & CTIO \\
4 & -165.143 & -129.545 & $\mathrm{~B}$ & 17.7746 & $\mathrm{I}$ & 17.1466 & CTIO \\
\hline \hline
\end{tabular}

${ }^{a}$ Coordinates are offsets from the center of the cluster

${ }^{b} \mathrm{~F} 5=\mathrm{F} 555 \mathrm{~W}, \mathrm{~F} 3=\mathrm{F} 336 \mathrm{~W}, \mathrm{~F} 2=\mathrm{F} 255 \mathrm{~W}$

Note. - The complete version of this table is in the electronic edition of the Journal. The printed edition contains only a sample. 
Table 3: New Horizontal Branch Star Table

\begin{tabular}{crrcrrrc}
\hline \hline Star & $\Delta \alpha{\text { (arcsec })^{\mathrm{a}}}^{\mathrm{a}}$ & $\Delta \delta$ (arcsec $^{\mathrm{a}}$ & Filter & Magnitude & Filter & Magnitude & Telescope \\
\hline 1 & -202.1681 & 382.3869 & $V$ & 15.8505 & $B$ & 15.7814 & CTIO \\
2 & -307.5504 & -253.5796 & $V$ & 16.2131 & $B$ & 16.1191 & CTIO \\
2 & -307.5504 & -253.5796 & $I$ & 16.4943 & & & CTIO \\
3 & 4.7069 & 1.1835 & $V$ & 14.5378 & $B$ & 14.2150 & HST \\
3 & 4.7069 & 1.1835 & F555W & 14.4650 & & & HST \\
\hline \hline
\end{tabular}

${ }^{a}$ Coordinates are offsets from the center of the cluster 


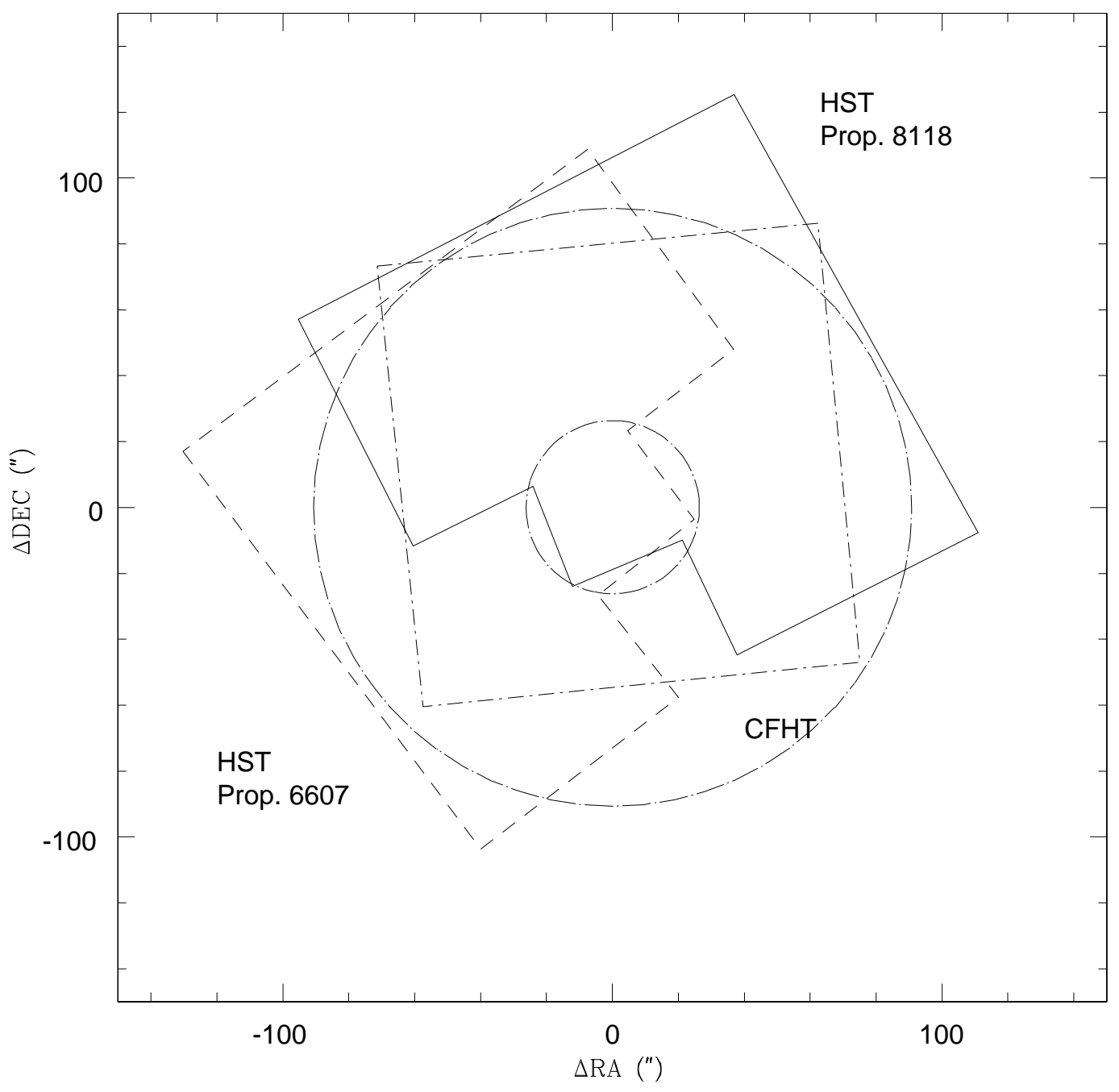

Fig. 1.- Fields observed in the core of M5. The inner circle represents the core radius $\left(r_{c}=26^{\prime \prime} .3\right)$ and the outer circle represents the half-mass radius $\left(r_{h}=90^{\prime \prime} \cdot 75\right)$. 

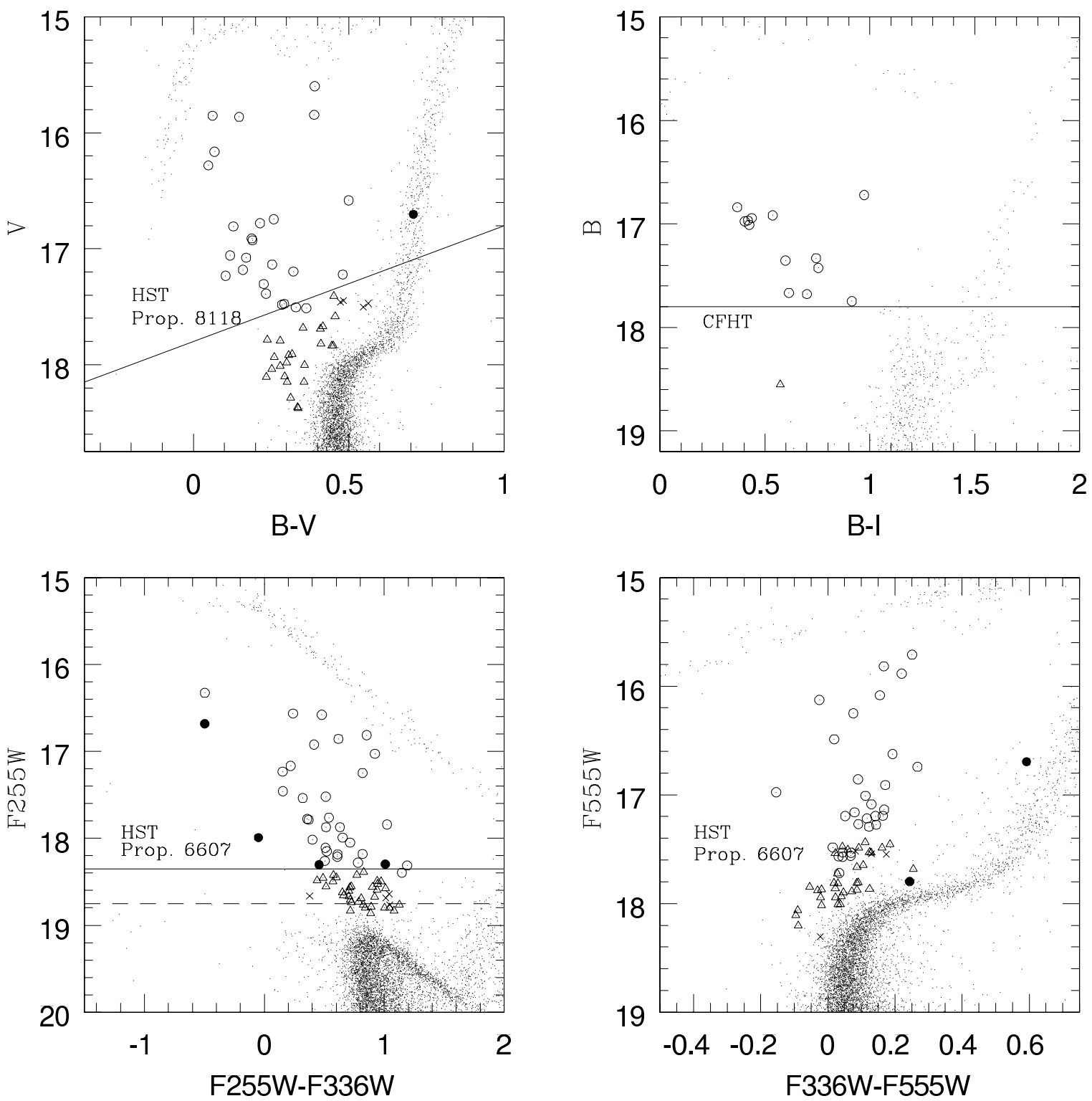

Fig. 2.- CMDs for the core datasets. BBSSs are shown as circles and FBSSs are shown as open triangles. Crosses mark FBSSs with $B-V>0.47$. Filled black circles are BBSSs that would have been selected if only the information from the $\left(m_{255}, m_{255}-m_{336}\right)$ CMD was used (see $\S 4.2)$. The solid line in the $\left(m_{255}, m_{255}-m_{336}\right)$ CMD corresponds to the BBSS cutoff at $m_{255}=18.35$ and the dashed line corresponds to the FBSS cutoff used by Ferraro et al. $(1997 \mathrm{~b})$ at $m_{255}=18.5$. The solid lines in the $(V, B-V)$ and $(B, B-I)$ CMDs represent the BBSS cut at $B=17.81$. Stars in the CFHT panel are only those falling outside the HST fields. 

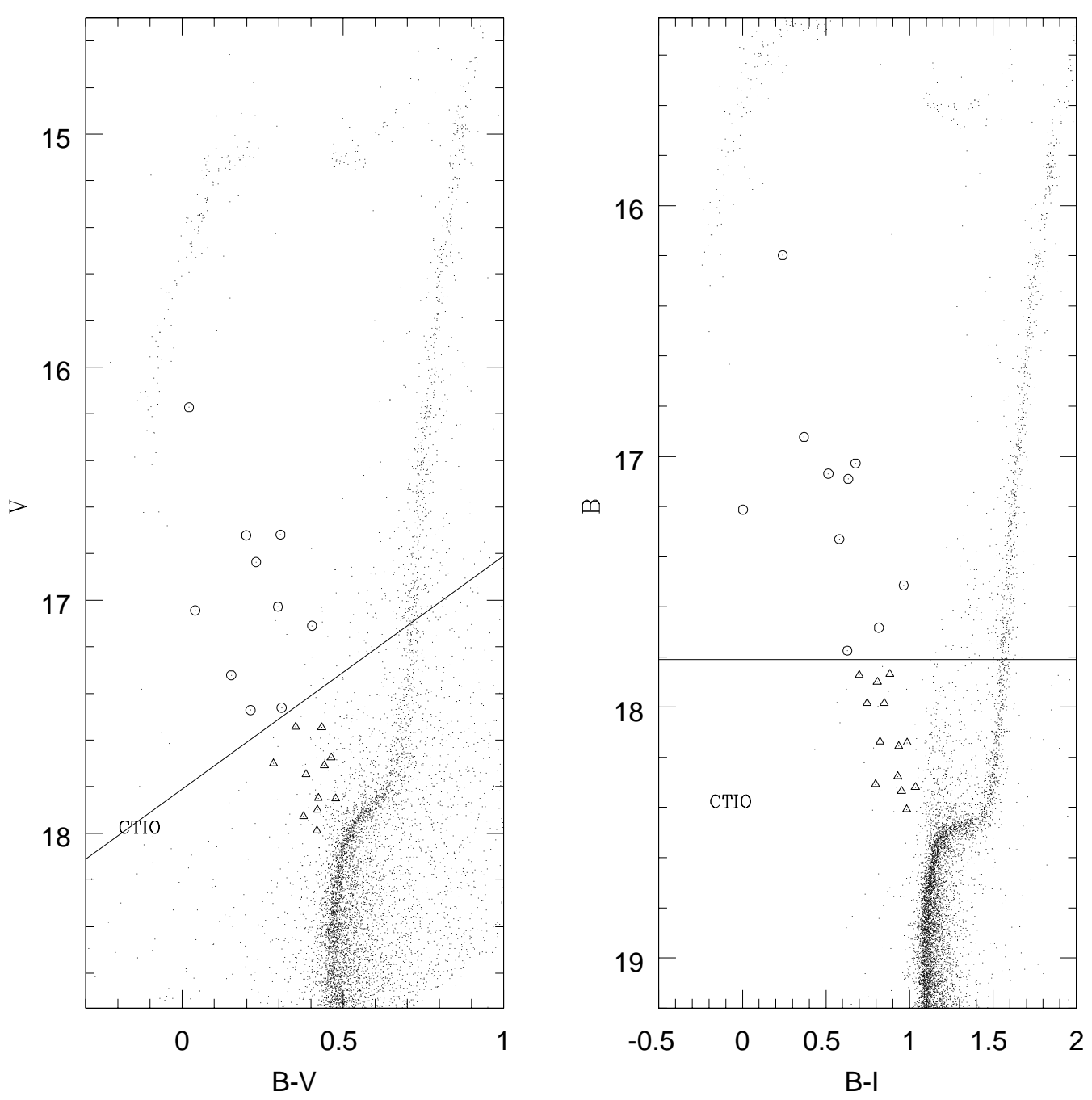

Fig. 3.- Ground-based CMDs for the area of the cluster not covered by the HST and CFHT fields. BBSSs are circled and FBSSs are triangles. 


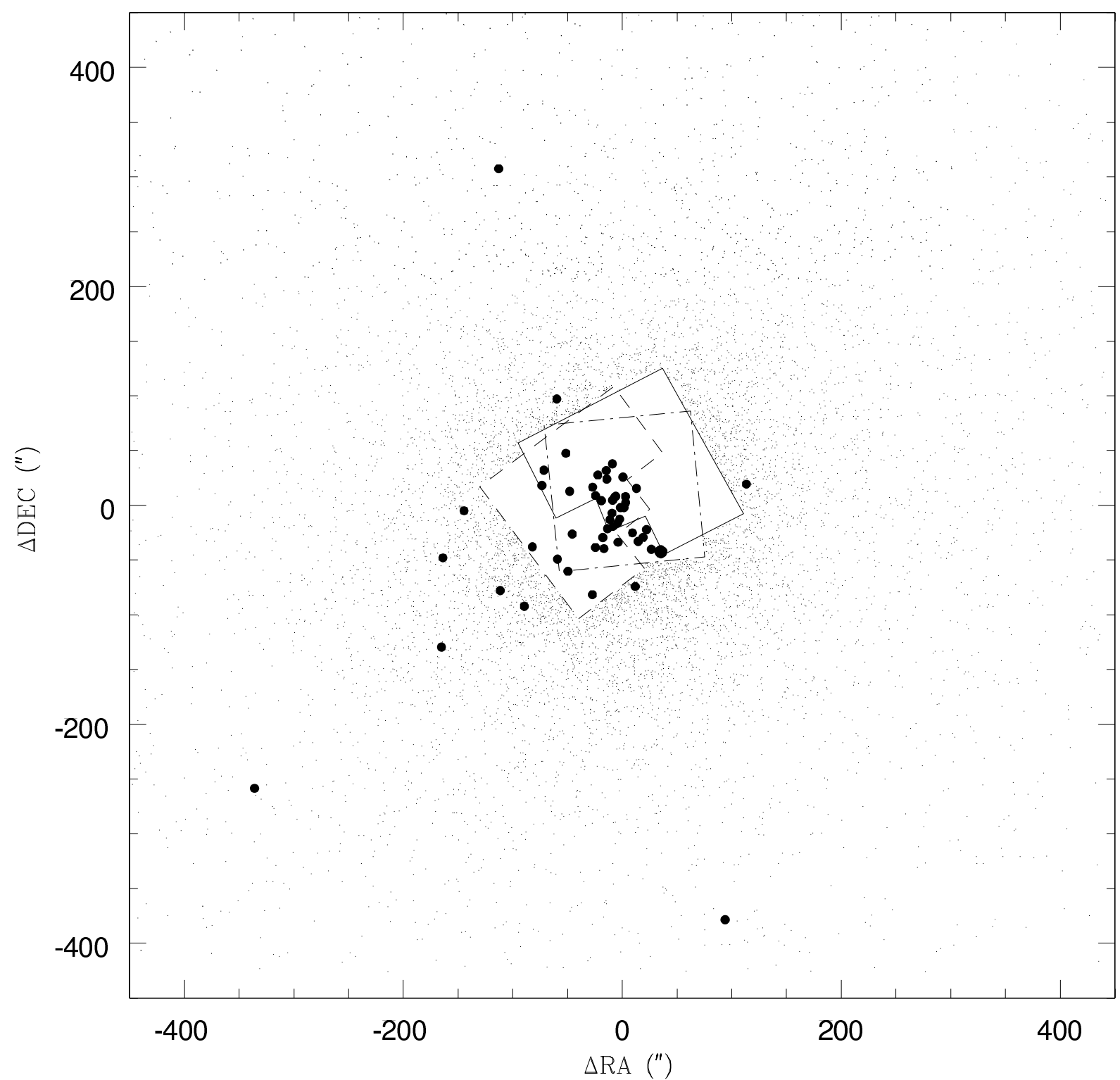

Fig. 4.- BBSSs using our limit cuts are large black circles. The non-BBSSs have been removed from the core for clarity. 

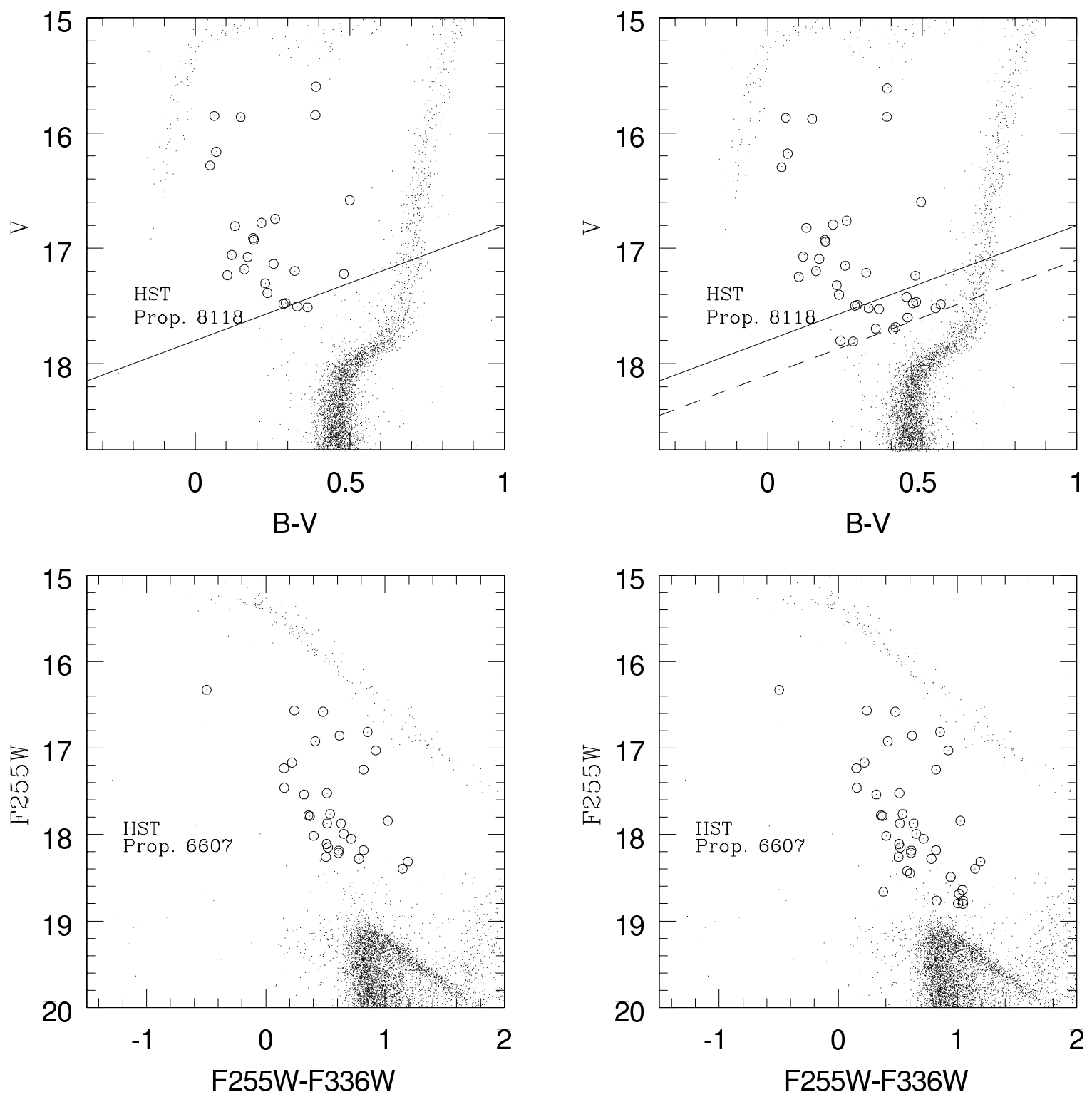

Fig. 5.- The CMDs on the left show the BBSS sample selected using the cut (solid line) proposed by us at $B=17.81$, while the CMDs on the right show the BBSS sample selected using the cut (dashed line) from Ferraro et al. (1997b) at $B=18.1$. We find better sample agreement in the upper and lower CMDs when the $B=17.81$ cut is used. 


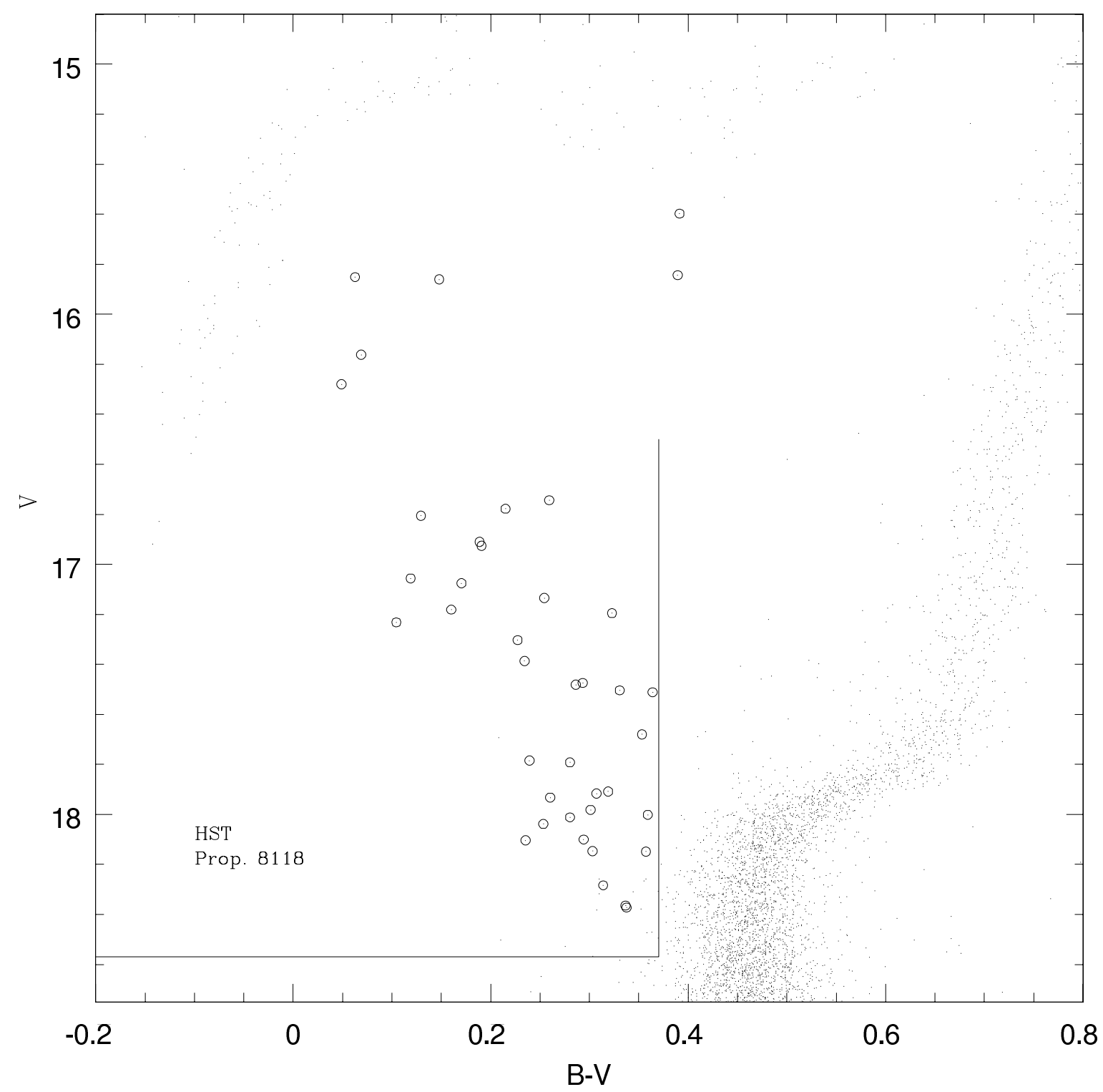

Fig. 6. - BSSs used for the Piotto et al. (2004) comparison are circled. 


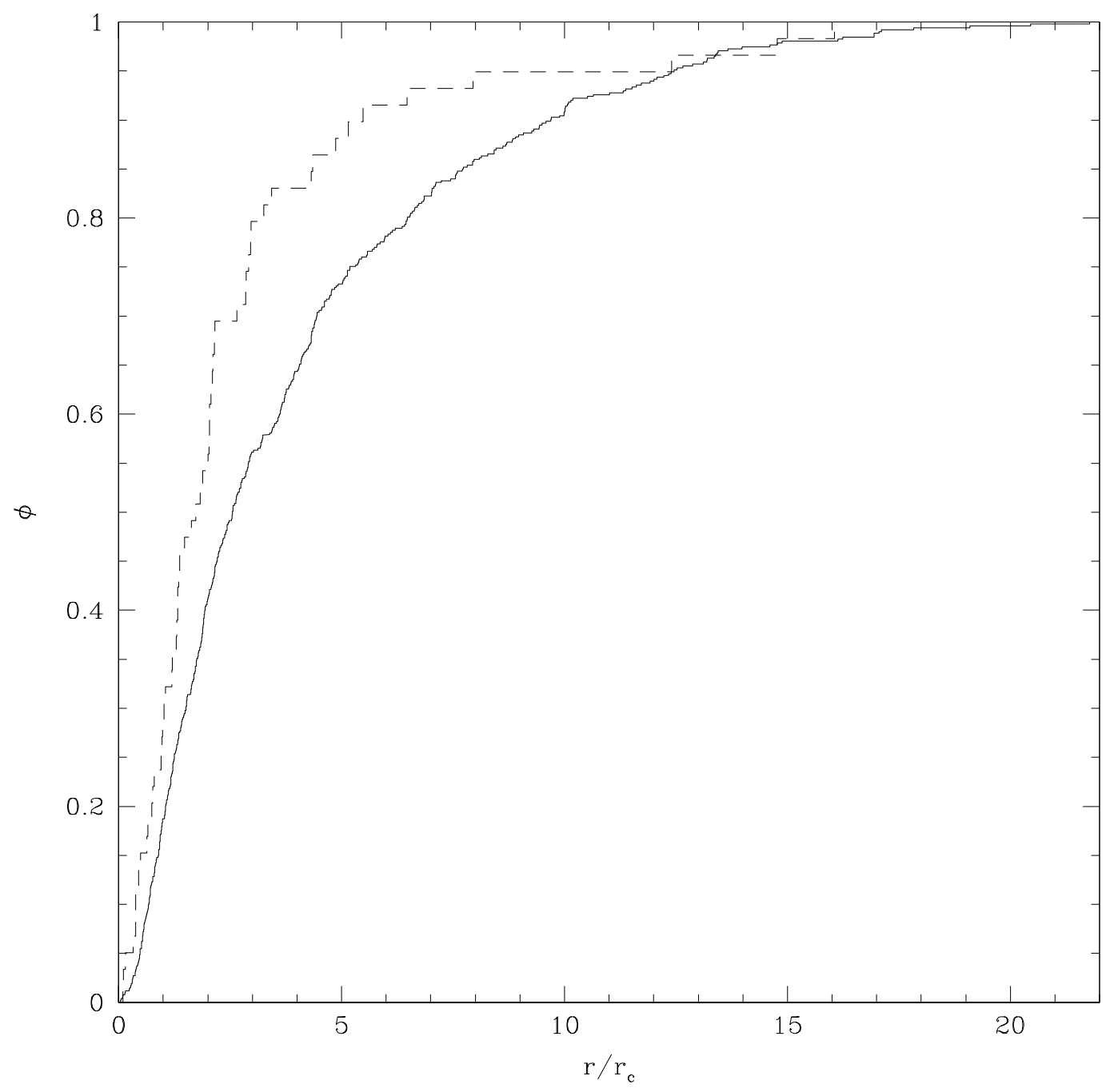

Fig. 7.- The cumulative radial distributions for the BBSSs (dashed line) and the HB stars (solid line) of M5. 


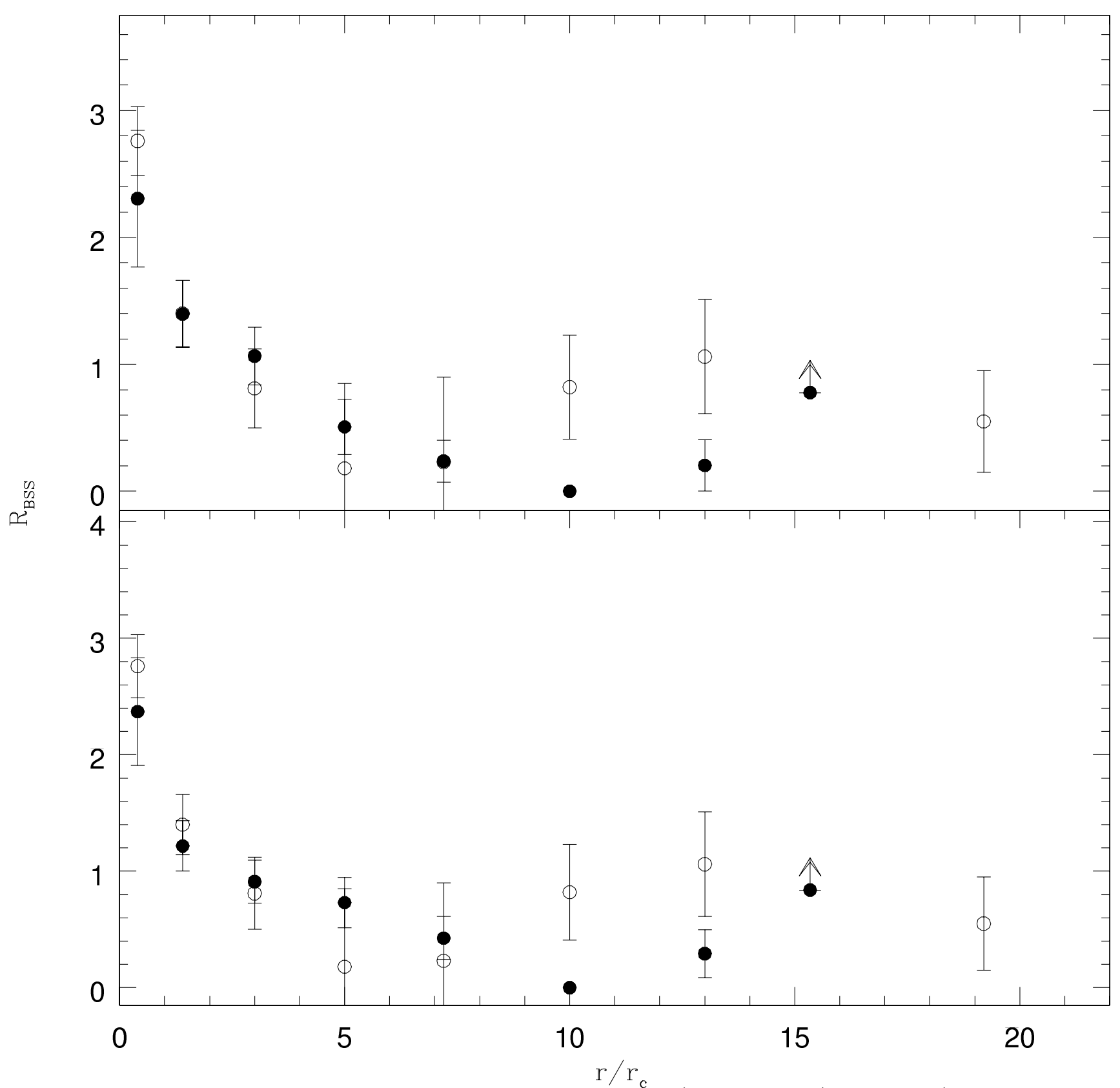

Fig. 8.- Radial distributions of blue stragglers for M5 (filled circles) and M3 (open circles; Ferraro et al. 1997b). The top panel shows the radial distribution for M5 when our BBSS cuts are used (see §4.1), while the bottom plot shows the radial distribution when the BBSS cuts of Ferraro et al. (1997b) are used. 


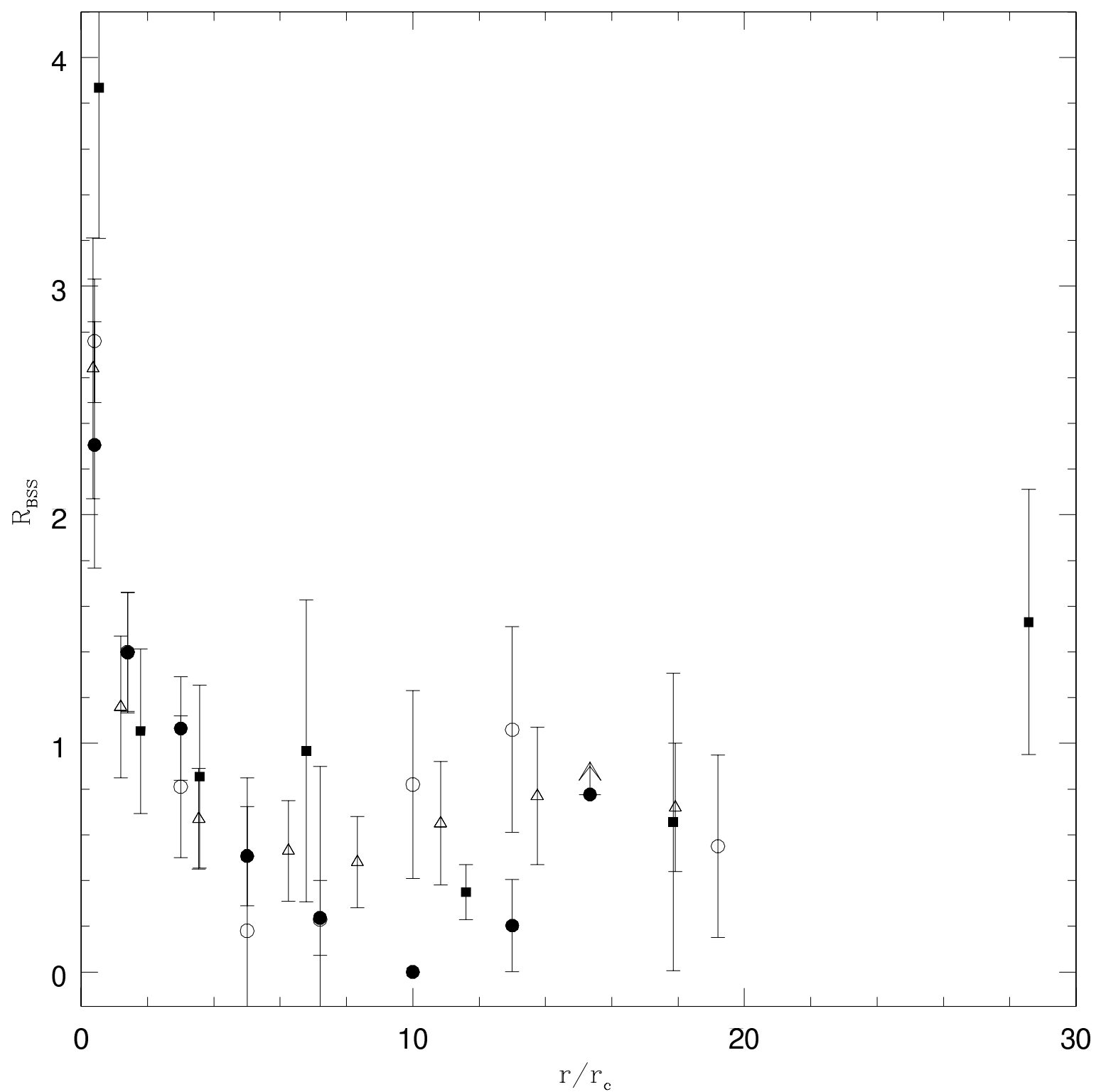

Fig. 9.- Radial distributions for M5, M3, 47 Tuc, and NGC 6752. Filled circles are data for M5, open circles are data taken from Ferraro et al. (1997b) for M3, open triangles are data taken from Ferraro et al. (2004) for 47 Tuc, and filled squares are data taken from Sabbi et al. (2004) for NGC 6752. 


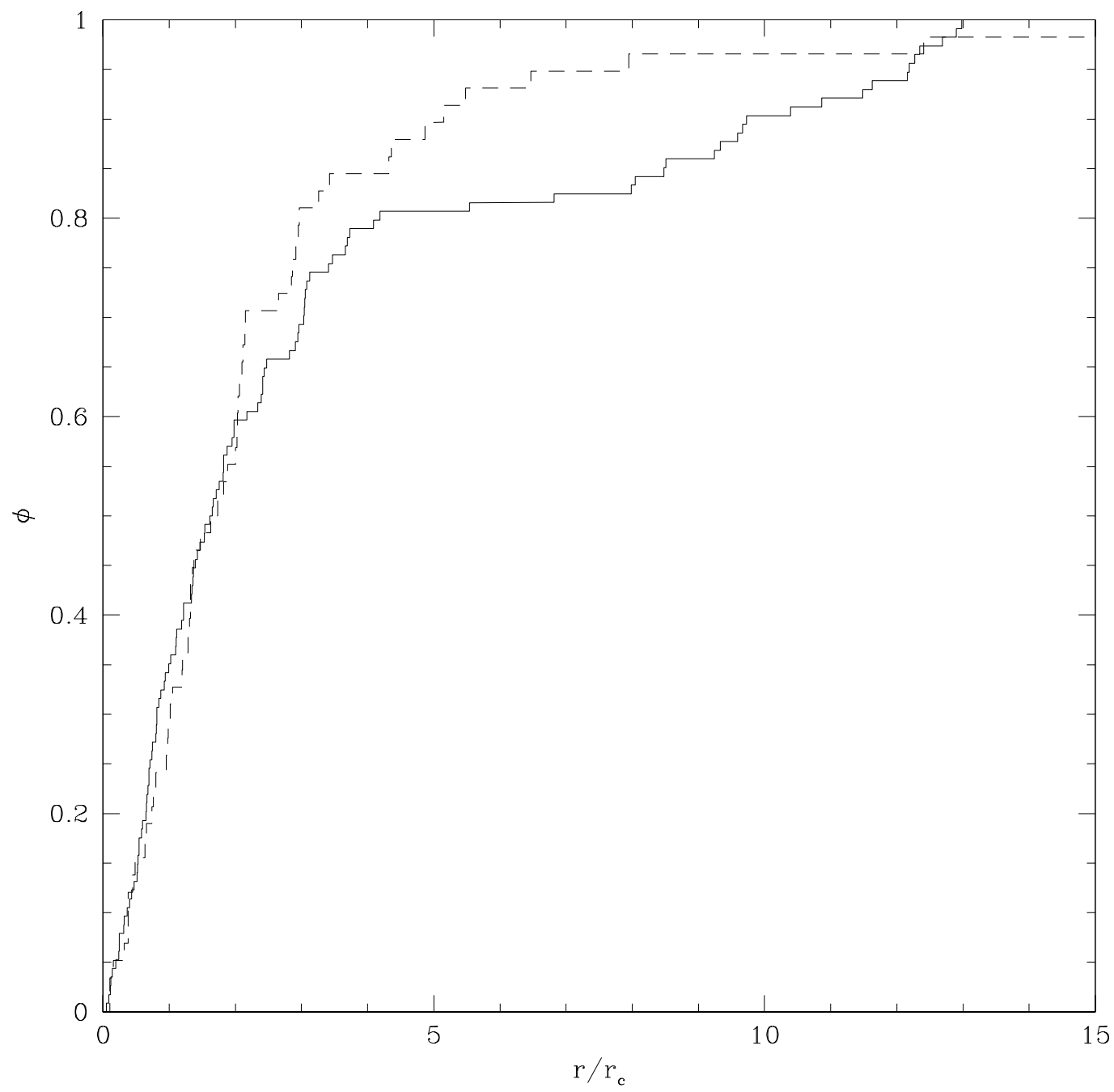

Fig. 10.- The cumulative radial distributions for M3 (solid line) and M5 (dashed line). The radial coordinates are scaled for the different core radii of the two clusters. 


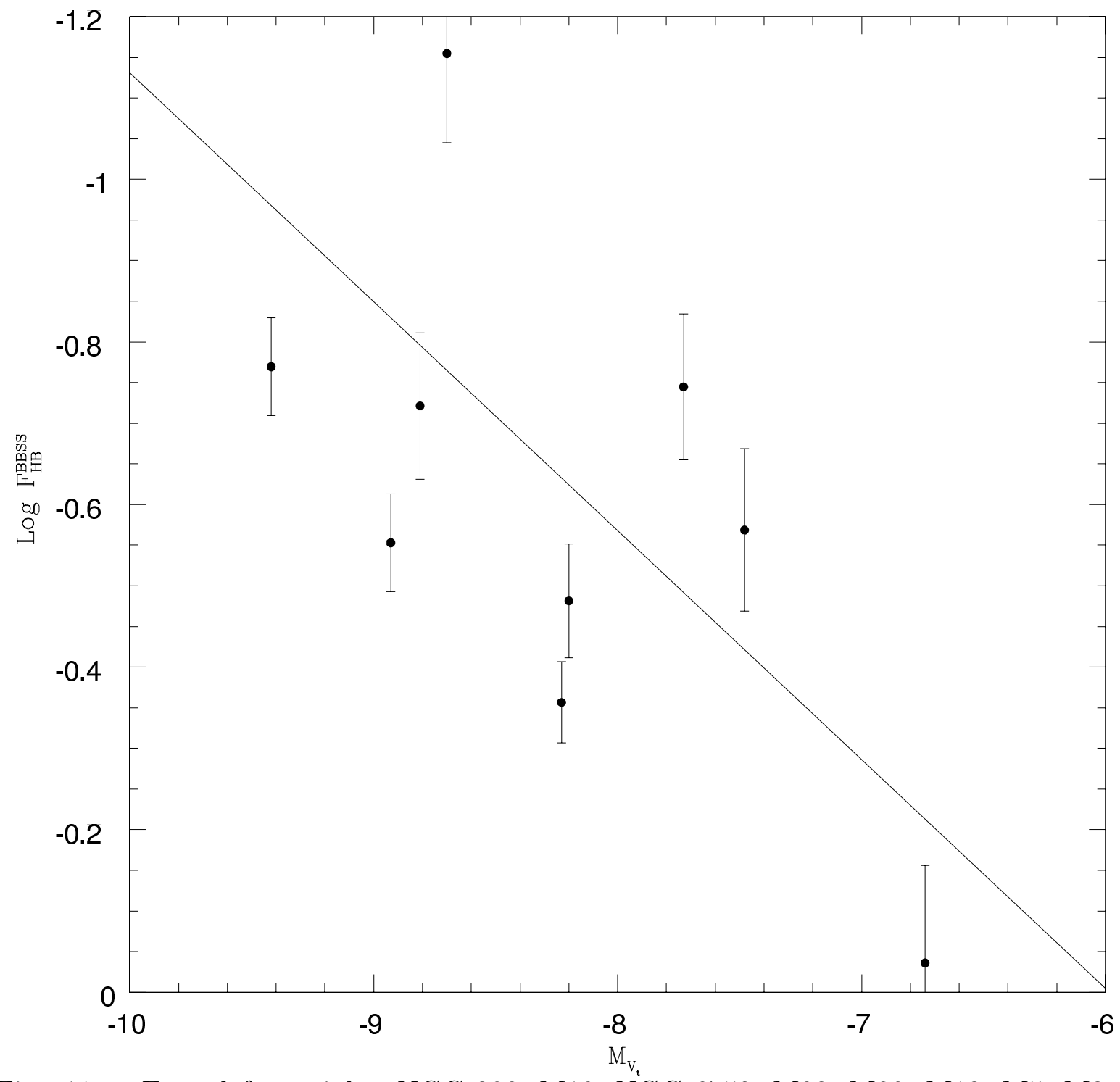

Fig. 11.- From left to right: NGC 288, M10, NGC 6752, M92, M80, M13, M5, M3, and 47 Tuc. The best fit line through the data points has an equation $y=0.28 x+1.68$ with an error in the slope of \pm 0.11 . 